del Mediterraneo Occidentale. Giorn. Bot. Ital., 103(6): 457-474.

GIACCONE, G., P. COLONNA, C. GRAZIANO, A.M. MANNINO, E. TORNATORE, M. CORMACI, G. FURNARI \& B. SCAMMANCCA - 1985- Revisione della flora marina di Sicilia e Isole minori. Boll. Acc. Gioenia Sci. Nat., 18(326): 537-781.

GONZ LEZ GARCэA, J.A. y F. CONDE POYALES -1993- Estudio biogeográfico de las Fucales y Laminariales atlánticas en el litoral mediterráneo de Marruecos. Acta Bot. Malacitana, 18: 39-44.

GONZ LEZ GARCэA, J.A. y F. CONDE POYALES -1994- Catálogo del macrofitobentos del Mediterráneo de Marruecos. Acta Bot. Malacitana, 19: 5-27.

IZQUIERDO, J.L., F. CONDE POYALES y A. FLORES MOYA -1995-Adiciones a la distribución geográfica del género Phyllariopsis Henry \& South (Laminariales, Fucophyceae) en la Península Ibérica e Islas Baleares. Acta Bot.
Malacitana, 20: 281-282.

PERRET-BOUDOURESQUE, M. \& H. SERIDI 1989-Inventaire des algues marines benthiques $d$ Algerie. Gis Posidonie publ., Marseille, Fr.

RIBERA, M.A., A. Gэ MEZ GARRETA, T. GALLARDO, M. CORMACI, G. FURNARI \& G. GIACCONE -1992- Check-list of Mediterranean Seaweeds. I. Fucophyceae (Warming, 1884). Botanica Marina, 35: 109130.

Aceptado para su publicación en Mayo de 1995

Dirección de los autores. J.A. GONZ LEZ GARCэA: Escuela Universitaria de Formación del Profesorado. 29805, Melilla. F. CONDE POYALES. Dpto. Biología Vegetal. Facultad de Ciencias. Universidad de Málaga. Apdo. 59. 29010, Málaga.

\title{
49. ADICIONES A LA FLORA ALICANTINA
}

\section{Ana JUAN, Luis SERRA y Manuel B. CRESPO}

Palabras clave. Corología, Espermatófitos, Taxonomía, Alicante, España.

Key words. Chorology, Spermatophyta, Taxonomy, Alicante, Spain.

Como resultado de las prospecciones realizadas en los últimos años en la provincia de Alicante se han descubierto algunas plantas que resultan destacables para el catálogo florístico de este territorio. Algunos de los taxones incluidos resultan novedades para la flora provincial y en cualquier caso todos ellos han sido hallados en localidades que constituyen ampliaciones considerables de sus áreas de distribución.

Para cada planta se aportan datos referentes a su comportamiento ecológico, fitosociológico y biogeográfico.

El material citado en el presente trabajo se encuentra depositado en el herbario $\mathrm{ABH}$ de la Universidad de Alicante (Holmgrem \&

\footnotetext{
* Trabajo financiado en parte a cargo del Proyecto PB91-0889 de la D.G.I.C.Y.T., y del convenio de investigación GV-0863/93, suscrito entre la Universidad de Alicante y la Consejería de Medio Ambiente de la Generalitat Valenciana.
} 
Holmgrem, 1993); también han sido consultados en algún caso los herbarios ALME, JAEN, K, MGC, MUB, VAB y VF (Holmgrem et al., 1990) para completar algunos datos corológicos.

Los datos biogeográficos y bioclimáticos siguen las directrices de lo reseñado en Rivas Martínez (1987), Alcaraz et al. (1991) y De la Torre et al. (en prensa).

Astragalus epiglottis L. subsp. asperulus (Dufour) Nyman

HS, ALICANTE: Millena, Port de Millena, 30SYH2890, 600 m, 13-V-1994, M. B. Crespo, L. Serra, A. Juan \& L. Rull, ABH 10701.

Taxón recientemente dado a conocer como novedad para Alicante (Serra \& Mateo, 1993: 151), cuya distribución hasta la fecha se circunscribía al cuadrante SW de la Península y NW de frica; además de una única localidad conocida en Valencia, de donde curiosamente fue descrita (Dufour, 1820: 295).

En ambas localidades alicantinas se presenta en el piso mesomediterráneo medio-inferior, bajo ombroclima seco-subhúmedo y en exposiciones soleadas, donde forma parte de pastizales terofíticos subnitrófilos de Taeniathero-Aegilopion geniculatae (Rivas Goday \& Rivas Mart. 1963) Rivas Mart. \& Izco 1977.

Caralluma munbyana (Decne) N. E. Br. subsp. hispanica (Coincy) M. B. Crespo \& Mateo, comb. \& stat. nov.

\& Boucerosia munbyana Decne var. hispanica Coincy, J. Bot. (Morot) 1898: 250. 1898.

$\& B$. hispanica (Coincy) Coincy, J. Bot. (Morot) 1899: 336. 1899.

HS, ALBACETE: $5 \mathrm{~km} \mathrm{NW}$ of Hellín, on road to Liétor, $600 \mathrm{~m}$, on hide ridges $\mathrm{N}$ of road, growing in dense clumps of Stipa gigantea, 11-XI-1981, Bruyns 2471, K.

HS, ALICANTE: Millena, Port de Millena, 30SYH2890, 600 m, 13-V-1994, M. B. Crespo, L. Serra, A. Juan \& L. Rull, ABH 10699. Finestrat, Serra de la Cortina, 30SYH4572, $500 \mathrm{~m}, 17-\mathrm{V}$ 1994, M. B. Crespo, L. Serra \& A. Juan, ABH 11566. Near Villajoyosa, Finestrat, $600 \mathrm{~m}$, on hillside behind mountain, with Quercus ilex, Chamaerops humilis, Sedum sediforme, Erica multiflora, 19-XI-1985, Bruyns 2473, K, NBG.

HS, MURCIA: Caravaca, Cerro de los Siete Peñones, 30SWH9517, $850 \mathrm{~m}$, grietas de rocas calizas, 13-VI-1982, C. Selma, MUB 20592. Ibidem, 500 m, Bruyns 2400, 8-XI-1985, K. Abanilla, Sierra del Cantón, ladera pedregosa, IX-1985, F. Alcaraz, MUB 13720. Ibidem, 30SXH7539, $700 \mathrm{~m}$, fisuras anchas de rocas, 24-IV-1982, MUB 8603.

Las poblaciones ibéricas de Caralluma munbyana presentan algunas diferencias morfológicas en los tallos, flores y frutos, que permiten diferenciarlas de las poblaciones típicas del norte de frica. Este hecho fue evidenciado por Coincy (1898), quien propuso inicialmente un taxón varietal (var. hispanica) que posteriormente elevó al rango específico (Coincy, 1899). No obstante y pese a lo indicado por Bruyns (1987), el aislamiento geográfico y las diferencias morfológicas existentes entre ambos apuntan mejor a un tratamiento intermedio, por lo que se propone aquí una nueva combinación en el rango subespecífico.

C. munbyana subsp. hispanica es, por tanto, un endemismo de área muy limitada, restringido hasta ahora a los subsectores Manchego-Murciano y Manchego-Espunense (provincia CastellanoMaestrazgo-Manchega), así como en el AlcoyanoDiánico (provincia Catalano-ValencianoProvenzal); subsector este último en el que ocupa las áreas de contacto con los territorios murcianoalmerienses (fig. 1).

Aparece tanto en el piso mesomediterráneo como en el termomediterráneo y siempre bajo ombroclima seco, dado que suele ocupar exposiciones bien soleadas.

En cuanto al comportamiento fitosociológico, algunos autores (Honrubia et al., 1983: 320) la han considerado típica del Rhamno-Quercetum cocciferae Br.-B1. \& O. Bolòs 1958, al menos en lo que se refiere a sus localidades murcianas (entre 700 y 800 m, en las sierras de Carrascoy, Almenara y Ricote).

Las primeras referencias de esta planta en la provincia de Alicante fueron ofrecidas por Bruyns (1987: 85). En las localidades alicantinas se encuentra en terrenos pedregosos con cierta pendiente, soleados. Siempre refugiada a la sombra de la vegetación o de las piedras, en los claros de comunidades de Stipion tenacissimae Rivas Mart. 1977. 


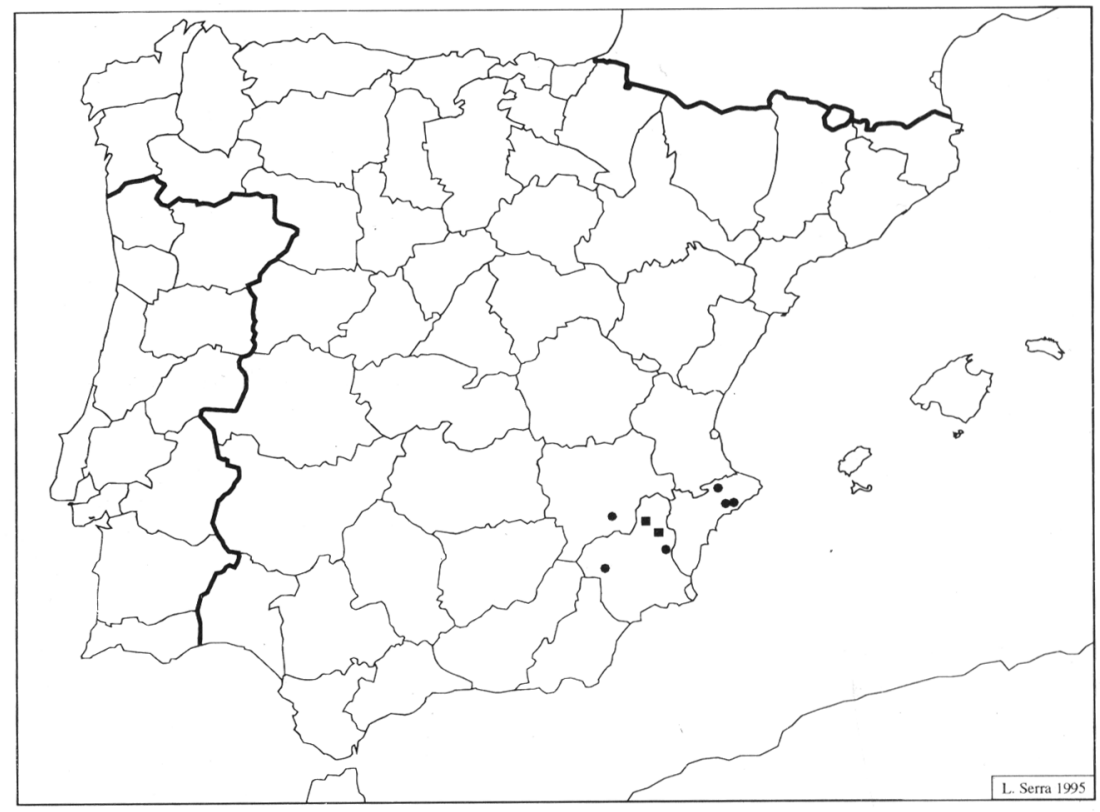

Figura 1. Distribución conocida de Caralluma munbyana subsp. hispanica. . Citas bibliográficas, , citas de herbario.

\section{Mercurialis $\times$ malinvaudii Sennen эmalinvaudi}

(M. annua L. $\times$ M. tomentosa $\mathrm{L}$.)

*HS, ALICANTE: Petrer, Sierra del Cid, 30SXH9760, 740 m, 24-V-1994, A. Juan, ABH 10442.

Nototaxón descrito por Sennen (1902: 375) a partir de material recolectado en los alrededores de Aude (Francia). Los caracteres de las plantas alicantinas son netamente intermedios entre los de sus parentales, pudiendo reconocerse fácilmente por su porte elevado, sufruticoso en la base, sus hojas verde-grisáceas laxamente tomentosas y conspicuamente pecioladas, sus estípulas membranaceas con margen hialino-escarioso, etc. Estos caracteres se ajustan plenamente a la descripción original del nototaxón.

En la localidad alicantina forma parte de comunidades de Andryalion ragusinae Rivas Goday \& Esteve 1972, sobre sustratos pedregosos móviles notablemente nitrificados.

Se trata de un nototaxón francamente raro en la flora valenciana, del cual sólo se tiene constancia de su presencia en la provincia de Castellón (Benicarló, El Puig, junto al Poblado Ibérico, 3-III1987, V. J. Arán, VAB 92/1795). No obstante, dada la amplia distribución de sus parentales en el territorio español es una planta que deberá buscarse.

Ononis viscosa L. subsp. subcordata (Cav.) Sirj. HS, ALICANTE: Oriola, carretera San Miguel de Salinas a Pilar de la Horadada, km 27, 30SXG9498, $60 \mathrm{~m}$, herbazales sobre terrenos arenosos, 27-III-1994, M. B. Crespo \& L. Serra, ABH 10712.

Atendiendo a los datos actuales se trata de su segunda localidad provincial, dado que previamente había sido indicada en el castillo de La Vall de Gallinera por Rigual (1984: 314, ut O. subcordata Cav. subsp. cavanillesii Pau \& Sennen), al norte de la provincia de Alicante, donde además convivía con la subsp. breviflora (DC.) Nyman.

En la nueva localidad, con la que se amplía considerablemente su distribución alicantina, se presenta en terrenos arenosos sueltos, formando parte de comunidades sabulícolas termomediterráneas deMaresio-Malcolmienion ramosissimae Rivas Mart. 1977.

Orchis mascula (L.) L. subsp. olbiensis (Reuter) Ascherson \& Graebner 
HS, ALICANTE: Petrer, Sierra del Cid, 30SXH9860, 920 m, 1-IV-1994, A. Juanet al., ABH 9482 .

Taxón conocido de diversas localidades alcoyano-diánicas del norte de Alicante, de donde fue dado a conocer hace algún tiempo por Molero \& Rovira (1981). La nueva población es la más meridional conocida en la provincia de Alicante y la primera situada en territorios ayorano-villenenses. Participa en lastonares mesomediterráneos de TheroBrachypodion retusi Br.-B1. 1925, en umbrías protegidas y bastante frescas, aunque bajo ombroclima seco.

+Pennisetum setaceum (Forssk.) Chiov.

HS, ALICANTE: Elx, El Ferriol, 30SYH0241, $150 \mathrm{~m}, 22-\mathrm{VII}-1992$, A. de la Torre \& M. B. Crespo, ABH 1750.

Interesante taxón originario de frica oriental del que sólo se conocía su presencia en El Campello (Crespo et al., 1990: 260) como única localidad alicantina.

La nueva población amplía considerablemente hacia el sur y el interior su distribución alicantina y confirma su naturalización y expansión en esta provincia. Participa en comunidades subnitrófilas de Bromo-Oryzopsion miliaceae O. Bolòs 1970 y Saturejo-Hyparrhenion hirtae O. Bolòs 1962, siempre en el piso termomediterráneo y bajo ombroclima semiárido.

\section{Reseda lanceolata Lag. subsp. lanceolata}

HS, *ALICANTE: Oriola, Sierra de Pujálvarez, 30SXH8106, $300 \mathrm{~m}$, matorrales secos, 3-IV-1993, L. Serra 3258, ABH-LS 3669.

HS, ALMER $\rightarrow$ A: Sierra de los Filabres (Purchena), 13-VIII-1974, Ginés López, VF5345. Aguadulce, taludes junto a la carretera, 17-III-1984, WF37, Mateo \& Lázaro, VAB 84/1603. Vera, 150 m, terrenos baldíos, 17-III-1984, Mateo, VAB 84/ 1602. Los Lobos, 200 m, 18-III-1985, Mateo \& Mateu, VAB 85/2421. Laujar, Sierra Nevada, 7VIII-1949, Jerónimo, ALME 2400. Loma de los Yesares (base Sur de Sierra Cabrera, cerca de Carboneras), yesos del Mioceno superior, 30SWF9198, 200-300 m, 14-V-1983, Lázaro, ALME 7317. Tabernas, carretera al repetidor de la $S$ ) Alhamilla, $410 \mathrm{~m}$, margas con yesos, cantos rodados, espartal, 15-IV-1993, Cabezudo \& Navas, MGC 35894.
HS, JAÉN: Baeza, Jarafe Nuevo a Cabezagorda, 550 m, 30SVG5091, 16-V-1993, Jimenez Zafra \& Frias, JAEN 931010. Vílches, Jarabancil, terreno silíceo, 720 m, 30SVH5730, 8IV-1989, Hervás, JAEN 893008. Andújar, Arroyo Salado, terreno margoso, $220 \mathrm{~m}, 30$ SVH0606, 3VII-1984, Cano, JAEN 844541. Jaén, a la Imora, borde de la carretera, $580 \mathrm{~m}, 30 \mathrm{SVG} 2782$, Aguilar, JAEN 821855. Jaén, Polígono del Valle, $470 \mathrm{~m}$, 30SVG3182, Aguilar, JAEN 821815. Jaén a Torrequebradilla km 8, $300 \mathrm{~m}, 30-\mathrm{V}-1981$, 30SVG3686.

HS, M LAGA: Maro, Monte de la Cueva, 11V-1984, Cabezudo \& Guerra, MGC 13463.

HS, MURCIA: Sierra de Altaona, sobre un talud margoso, próximo a un camino, 28-V-1986, Ros Izquierdo, MUB 21130. Puerto de la Cadena, 30-IV-1986, Alcaraz, MUB 18128.

Interesante novedad para la flora alicantina, que hasta el momento sólo se conocía de las vecinas provincias de $\mathrm{Al}, \mathrm{Gr}$, Ma y Mu (Valdés-Bermejo, 1993: 464). Con la nueva localidad se amplía considerablemente el área de distribución de este taxón, siendo a su vez su localidad más septentrional conocida (fig. 2). Se presenta en matorrales seriales de Anthyllidetalia terniflorae Rivas Goday et al. in Rivas Goday \& Borja 1961, bastante degradados por factores antrópicos (pastoreo, remoción de terrenos, etc.), donde aparece acompañado de Astragalus alopecuroides subsp. grosii (Pau) Rivas Goday \& Rivas Mart., entre otros.

Seseli montanum L. subsp. granatense (Willk.) Pardo $\equiv$ Seseli granatense Willk.

HS, *ALICANTE: Benifato, Serra d Aitana, pr. base militar, 30SYH3881, 1550 m, 15-IX-1992, L. Serra, ABH 2304.

HS, *ALBACETE: Calar de Socovos, 30SWH8340, $1290 \mathrm{~m}$, rellanos de rocas calizas, 8XI-1984, F. Alcaraz \& P. Sánchez Gómez, MUB 22105. Letur, Carrasca Gorda, pedregales calizos, 28-X-1988, P. Sánchez Gómez, MUB 27286.

HS, GRANADA: El Dornajo, Sierra Nevada, fisuras de rocas dolomíticas, 19-X-1979, M. Ladero, López Guadalupe \& J. Molero Mesa, MUB 1820. Sierra Nevada, région alpine, au Dornajo, VIII1851, Bourgeau 1197, K.

HS, MURCIA: Sierra de Moratalla, 30SWH6412, $1900 \mathrm{~m}$, suelo pedregoso-calizodolomítico, C. Selma, 1-XI-1984, MUB 22380. 


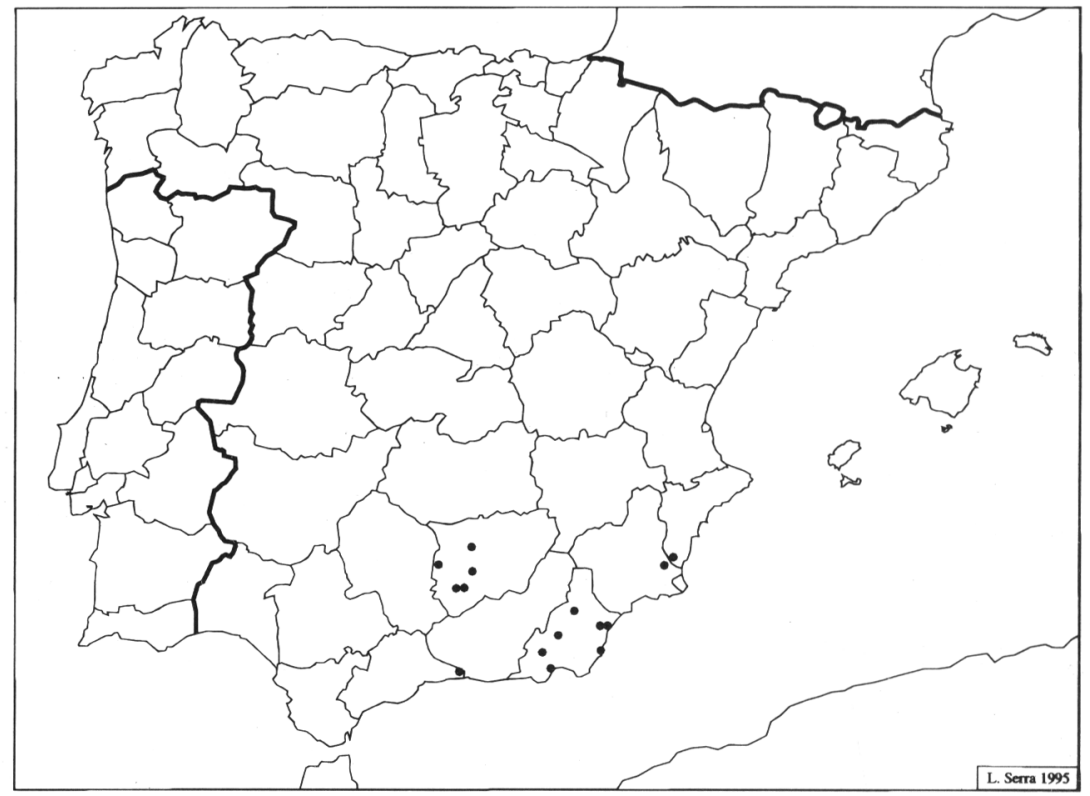

Figura 2. Distribución conocida de Reseda lanceolata subsp. lanceolata.

Moratalla, Revolcadores, 1950 m, 27-VII-1984, A. Robledo et al., MUB 7713. Moratalla, Sierra de la Muela, 30SWH9034, $1300 \mathrm{~m}$, rellanos de rocas calizas, 30-X-1982, P. Sánchez Gómez, MUB 22104.

Rigual (1984: 325) indicó Seseli montanum subsp. nanum (Dufour) O. Bolòs \& Vigo en la Serra d Aitana. Sin embargo este taxón es considerado como endemismo pirenaico y orocantábrico (Pardo, 1981: 175), diferenciándose de la subsp. granatense, entre otros caracteres, por no presentar pubescencia en el tallo y por tener estilopodios de color ocre. El material recolectado por nosotros en Aitana se ajusta bien a los caracteres de la subsp. granatense, lo que biogeográficamente resulta más coherente con la distribución conocida del grupo.

Por otra parte, S. montanum subsp. granatense era considerado hasta ahora como un endemismo bético (Pardo, op. cit.; Rivas Martínez et al., 1991: 60); pero recientemente ha sido indicado de diversos puntos de Murcia: sierras del Carche (Molero, 1985: 154), Revolcadores (Selma, 1992: 99) y Segura (Sánchez \& Alcaraz, 1993: 203). Así pues la localidad alicantina debe considerarse como la población más septentrional conocida de este taxón (Fig. 3).

Según lo indicado, $S$. montanum subsp. granatense deberá considerarse como una planta de óptimo en los pisos supra y oromediterráneo de la provincia Bética, con irradicaciones hacia las altas montañas de los subsectores Manchego-Murciano (provincia Castellano-Maestrazgo-Manchega) y Alcoyano-Diánico (provincia Catalano-ValencianoProvenzal).

Desde el punto de vista fitosociológico puede considerarse como una característica de las alianzas Minuartio-Poion ligulatae O. Bolòs 1962 -dando nombre al Seseli-Festucetum hystricis Mart. Parras, Peinado \& Alcaraz 1987 bético-, y XeroacanthoErinaceion anthyllidis (Qúezel 1953) O. Bolòs 1967 (Astragalo-Festucetum hystricis Quézel 1953, Saturejo-Velletum spinosae Rivas Goday 1968 corr. Alcaraz et al. 1991 y Erinaceo-Genistetum longipedis O. Bolòs \& Rigual in O. Bolòs 1967).

En la Sierra de Aitana se encuentra en las zonas culminales venteadas (entre 1500-1550 m), entre las grietas de los roquedos calizos y las grandes matas de Erinacea anthyllis Link, Genista longipes Pau y Vella spinosa Boiss., en el seno de la comunidad Erinaceo-Genistetum longipedis.

Es, por tanto, uno más de los taxones béticos que alcanzan las altas sierras alcoyano-diánicas. 


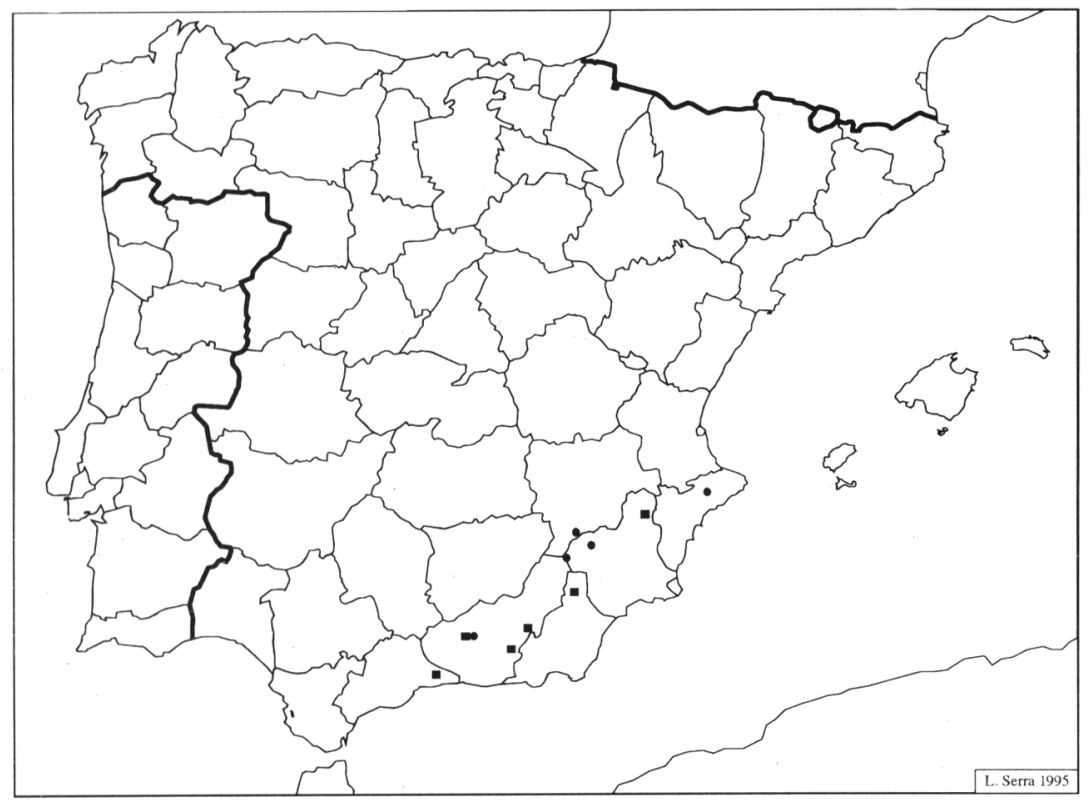

Figura 3. Distribución conocida de Seseli montanum subsp. granatense. Citas bibliográficas, citas de herbario.

AGRADECIMIENTOS. Agradecemos a los conservadores de los diferentes herbarios el préstamo de algunos de los materiales citados. Una parte de este trabajo fue realizada gracias a una beca concedida por Bancaja a unos de los autores (Luis Serra) para una estancia en los Royal Botanic Gardens, Kew.

\section{BIBLIOGRAFÍA}

ALCARAZ, F., P. SÁNCHEZ-GÓMEZ y A. DE LA TORRE -1991- Biogeografía de la provincia Murciano-Almeriense hasta el nivel de subsector. Rivasgodaya, 6: 77-100.

BRUYNS, P.V. -1987- Miscellaneous notes on Stapeliae (Asclepiadaceae). Bradleya, 5: 77-90.

COINCY, A. DE -1898- Plantes nouvelles de la flore d'Espagne ( $8^{\mathrm{e}}$ note). J.Bot. (Morot), 1898: 250-252.

COINCY, A. DE -1899- Plantes nouvelles de la flore d'Espagne ( $10^{\mathrm{e}}$ note. Fin). J.Bot. (Morot), 1898: 332-338.

CRESPO, M.B., M.L. MANSO y G. MATEO 1990- Pennisetum setaceum (Poaceae) especie nueva para el continente europeo. Anales Jard.
Bot. Madrid, 47(1): 260.

DE LA TORRE, A., F. ALCARAZ y M. B. CRESPO

- en prensa - Aproximación a la biogeografía del sector Setabense (provincia CatalanoValenciano-Provenzal). Rivasgodaya.,

DUFOUR, M.L. -1820-Coup d'oil topographique sur le ville de Xativa et sur Moxente, dans le royaume de Valence, et bouquet botanique de leurs environs. Ann. Gen. Sci. Phys. Bruxelles, 7: 281-310.

HOLMGREM, P.K. \& N.H. HOLMGREM -1993Additions to Index Herbariorum (Herbaria), edition 8 - Second Series. Taxon, 42: 489-505.

HOLMGREM, P.K., N.H. HOLMGREM \& L.C. BARNETT -1990- Index Herbariorum. Part I. The Herbaria of the world. Ed. 8. Regnum Veg., 120.

HONRUBIA, M., F. ALCARAZ, E. GRACIA y X. LLIMONA -1983- El componente fúngico de las principales comunidades vegetales del $\mathrm{SE}$ de España. Lazaroa, 4: 313-325.

MOLERO, J. y A. M. ROVIRA -1981- De Flora Dianicae. Anales Jard. Bot. Madrid, 38(1): 303305.

MOLERO, J. -1985-Aportaciones a la flora del 
sudeste ibérico. Collect. Bot. (Barcelona), 16(1): 149-160.

PARDO, C. -1981- Estudio sistemático del género

Seseli L. (Umbelliferae) en la Península Ibérica. Lazaroa, 3: 163-188.

RIGUAL, A. -1984- Flora y vegetación de la provincia de Alicante. El paisaje vegetal alicantino. Instituto de estudios Juan Gil-Albert. Alicante.

RIVAS-MARTÍNEZ, S. -1987-Memoria del mapa de series de vegetación de España 1: 400.000. Publ. ICONA. Madrid.

RIVAS-MARTÍNEZ, S., A. ASENSI, J. MOLERO MESA, y F. VALLE -1991- Endemismos vasculares de Andalucía. Rivasgodaya 6: 5-76.

SÁNCHEZ GÓMEZ, P. y F. ALCARAZ -1993Flora, vegetación y paisaje vegetal de las Sierras de Segura Orientales. Inst. Est. Albacetenses.

SELMA FERRÁNDEZ, C. -1992- Fragmenta Chorologica Occidentalia, 4180-4189. Anales Jard. Bot. Madrid, 50(1): 96-100.
SENNEN, F. -1902- Herborisations aux environs de la Nouvelle (Aude), I. Bull. Soc. Bot. France, 49: 364-377.

SERRA, L. \& G. MATEO -1993- Sobre la distribución peninsular de Astragalus epiglottis L. subsp. asperulus (Dufour) Nyman. Collect. Bot. (Barcelona), 22: 151-153.

VALDÉS-BERMEJO, E. -1993-Reseda L. In: Castroviejo, S. et al. (Eds.), Flora iberica. 4. Cruciferae-Monotropaceae. Real Jardín Botánico, CSIC. Madrid.

Aceptado para su publicación en Julio de 1995

Dirección de los autores. Departamento de Ciencias Ambientales y Recursos Naturales (Botánica), Universidad de Alicante. Apartado 99. E-03080 Alicante. 